\title{
Assessment of dietary pattern and factors that affect hemoglobin concentration of third trimester pregnant women at Ambo Governmental Health Institutions, Ethiopia.
}

\author{
Kefiyalew $\mathbf{J}^{\mathbf{1}}$, Eshetu $\mathbf{G}^{\mathbf{1}}$ \\ Kotebe Metropolitan University, Addis Ababa, Ethiopia
}

\begin{abstract}
Introduction: Iron deficiency and anemia resulting from iron deficiency is considered to be one of the top ten contributors to the global burden of disease. More than half of the pregnant women in the world suffer from anemia and it contributes to $40 \%$ of maternal death worldwide. The aim of this study was to assess the dietary pattern and factors that affect hemoglobin concentration of third trimester pregnant women.
\end{abstract}

Methods: A cross-sectional study design and convenient sampling technique were used. 123 third trimester pregnant women were interviewed to assess their socio-demographic characteristics, dietary pattern, and clinical conditions. The hemoglobin values of the women were determined using Hemo Cue. Descriptive analysis and simple linear regression were used.

Results: About $35.8 \%$ of the pregnant women were anemic. The mean age [years] of the pregnant women was $25.8 \pm 5.4$ [SD] and 55.3\% were multiparous. The most commonly consumed food by the subjects was cereals $[98.4 \%]$ and coffee was found to be the most commonly consumed drink [93.5\%]. Majority of the pregnant women [57.8\%] had three meals a day. Maternal factors such as, dietary diversity score $[\beta=0.517, p=0.001]$ and level of education $[\beta=0.464,95 \%$ CI: $0.241,687$, $p=0.001]$ had positive linear relationship with hemoglobin. Whereas, factors such as the number of cups of tea taken per day $[\beta=-0.476,95 \%$ CI: $-.792,-0.161, p=0.003]$, consumption of legumes $[\beta=-0.813, p=0.001]$ and roots and tubers $[\beta=-\mathbf{0 . 8 2 8}, p=0.001]$ were negatively associated with maternal hemoglobin concentration. The adj. $\mathrm{R} 2$ is 0.396 indicating that these factors explain $39.6 \%$ of the variation in hemoglobin concentration of the study participants.

Conclusion: The dietary pattern and nutritional status of third trimester pregnant women were associated with maternal hemoglobin concentration.

Keywords: Third trimester pregnant women, Hemoglobin concentration, Dietary habit.

Abbreviations: WFP: World Food Program, WHO: World Health Organization, ANC: Antenatal Care, EDTA: Ethylene Diamine Tetra-acetic Acid, SPSS: Statistical Package for Social Science Students, ASF: Animal Source Food, DDS: Dietary Diversity Score.

Accepted on November $20^{\text {th }}, 2018$

\section{Introduction}

Anemia is a widespread public health problem associated with an increased risk of morbidity and mortality, especially in pregnant women and young children. It is a disease with multiple causes, both nutritional (vitamin and mineral deficiencies) and non-nutritional [infection] that frequently co-occur. It is assumed that one of the most common contributing factors is iron deficiency, and anemia resulting from iron deficiency is considered to be one of the top ten contributors to the global burden of disease [1].

Anemia is directly or indirectly responsible for $40 \%$ of maternal deaths. There is 8 to 10 fold increase in MMR when the Hb falls below $5 \mathrm{gm} / \mathrm{dl}$ [2]. Nearly half the pregnant women suffer from varying degree of anemia which also has the highest number of maternal deaths with the highest frequency of anemia related to poor dietary habits especially poor iron intake [3]. Inadequate dietary iron, foliate intake due to low vegetable consumption, perhaps low B12 intake and poor bioavailability of dietary iron from the fiber, phytate rich diets may be responsible for high prevalence of anemia in developing countries. Increased requirement of iron during growth and pregnancy and chronic blood loss contribute to higher prevalence in specific groups [2].

Human eating behavior depends on biological and cultural behaviors. Perceptions and food taboos are some factors that can affect the eating pattern of pregnant women. Cereal products, legumes and nuts contain iron and zinc inhabitants like 5 and 6 phospho inositol [4]. Hence, diets largely contain cereals are not good source of iron and eventually they can impair bioavailability of iron [5]. On the other hand, consumption of foods rich in vitamin $\mathrm{A}$ is crucial because women with low serum vitamin $\mathrm{A}$ are 1.8 times at greater risk of acquiring anemia compared to women who are not vitamin A deficient [6].

In addition, maternal socio-demographic characteristics, dietary pattern and nutrient intake could be important indicators of maternal anemia [7-13].

On the other hand, deficiency of micronutrients during intrauterine period is closely related to morbidity and mortality of the newborn $[7,14,15]$. Thus, pregnant women have increased 
Citation: Assessment of dietary pattern and factors that affect hemoglobin concentration of third trimester pregnant women at Ambo Governmental Health institutions, Ethiopia. 2018;2(2):36-42

demand of micronutrients and they are crucial for the health of the mother and the developing fetus [16].

Iron deficiency is the most common nutritional deficiency in pregnancy and has an important impact on maternal and fetal morbidity and mortality. Although the sign of anemia is not manifested, pregnant women at any stage have iron deficiency anemia. Maternal iron deficiency during pregnancy induces anemia in developing fetus; however, the severity tends to be less than in the mother. This is because placental iron transporter proteins are up regulated in maternal iron deficiency resulting in an increased efficiency of iron influx and a consequent minimization of fetal anemia [17].

The $\mathrm{Hb}$ values of pregnant women fall gradually during the middle and last gestational ages [18] with the highest prevalence of severe anemia being among third trimester pregnant women. It is imperative to consider that anemia prevalence advances greater as the gestation increases $[19,20]$.

If pregnancy occurs during adolescence, anemia not only increases maternal morbidity and mortality, but increases the incidence of poor birth outcomes in infant [i.e., low birth weight and prematurity] and also negatively impact iron status. Maternal under-nutrition [inadequate dietary intake] is significantly linked with infant low birth weight, due to preterm birth or intrauterine growth restriction, an underlying factor in $60-80 \%$ of neonatal deaths [21]. Low maternal body mass index is associated with intrauterine growth restriction and low birth weight.

Moreover, maternal anemia is associated with poor intrauterine growth and increased risk of preterm births and low birth weight rates. This in turn results in higher prenatal morbidity and mortality and higher infant mortality rate. A doubling of low birth weight rate and 2 to 3 -fold increase in the prenatal mortality rate was seen when the $\mathrm{Hb}$ is $<8 \mathrm{~g} / \mathrm{dl}$. Parental height and maternal weight are determinants of intrauterine growth and birth weight. Thus, maternal anemia contributes to intergenerational cycle of poor growth and birth weight [2]. Thus, ensuring adequate dietary intake during pregnancy is crucial.

Although pregnant women in the third trimester relied on a monotonous diet in Ethiopia, prevalence of inadequate iron intake was surprisingly low [4\%] [21]. However, the prevalence of anemia is high $[8,9,11]$.

Generally, the prevalence of anemia is becoming the major public health concern than before in developing countries like Ethiopia. Therefore, to prevent such nutritional deficiencies problem solving studies and implementation of different costeffective strategies are crucial.

\section{Materials and Methods}

A cross-sectional study design and convenient sampling method were used since the number of the women attending antenatal clinic at a point of time was limited. The study subjects were third trimester pregnant women who attended antenatal clinic at Ambo hospital and Ambo health centers of Ethiopia during the study period. The sample size of the study was dependent on the number of women who attended the ANC during third trimester. Hence, 123 subjects were interviewed for their dietary habit and their hemoglobin level was determined using HemoCue; a non dilution photometric portable device.

\section{Data collection process}

The data were collected by the researcher, nurses and laboratory technicians.

\section{Socio-demographic characteristics}

The study subjects were interviewed using pre-structured English questionnaire translated in to local language, Afan Oromo; and all the personal details, environmental conditions and access to mass media were obtained by the researcher and nurses at ANC clinic.

\section{Dietary habits}

The study subjects were interviewed for their food habit using twenty-four-hour recall and two weeks food frequency questionnaire both by the researcher and nurses at ANC clinic.

\section{Recording pregnancy complications}

Signs of pregnancy complications like swelling of hands and feet, blurring of vision, breathlessness during normal exercise, high colored urine, headaches, and fever were recorded through interview and vital signs observation. Hypertension was also ruled out using blood pressure apparatus and stethoscope.

\section{Blood sample collection for hemoglobin determination}

The most common sites used were the antecubital fossa of the arm. All the necessary materials and equipment [sterile syringe and needle, protective glove, tourniquet, gauze pads or cotton, $70 \%$ alcohol, EDTA anti-coagulated test tubes and labeling markers] were assembled and two [2 $\mathrm{ml}$ ] of venous blood samples were collected both by the researcher and nurses in EDTA anti-coagulated vacutainer test tubes for each client (Figure 1).

\section{Biochemical analysis}

\section{Determination of Hemoglobin: HemoCue non-dilution photometric technique}

A small drop [10 $\mu 1]$ of blood was drawn by capillary attraction [only one test at a time] into a specially designed single used micro-cuvette of only $0.13 \mathrm{~mm}$ light-path which contains dry reagents [sodium desoxycholate, sodium azide, and sodium nitrite]. These lyse the blood and convert it to azidemethemoglobin, the absorption of which is read electronically in the HemoCue meter at wavelengths $565 \mathrm{~nm}$ and $880 \mathrm{~nm}$ [later reading compensates for any turbidity in the sample]. The cuvettes were not reused. A direct read-out of hemoglobin values [ $\mathrm{g} / \mathrm{dl}]$ were obtained within 1 minute of inserting the cuvette in the meter. The same technique for all subjects was used to keep the consistency of the results.

\section{Data analysis}

After completion of the data collection, laboratory investigation and careful data quality assurance the information obtained from each client were entered into SPSS [statistical package for social science students] version 20 . The statistical analyses were performed using parametric tests. 
I. Descriptive statistics was used to present the percentage, frequency, mean and the standard deviation of the occurrence of events for socio-demographic characteristics, dietary pattern and anemia status.

II. Multiple regression analysis was a model used to study the relationship between the outcome variables and the independent variables.

\section{Results}

The socio-demographic characteristics of the third trimester pregnant women $[\mathrm{n}=123]$ is described in Table 1 . The mean age of the pregnant women was $25.8 \pm 5.4$ and the mean family size was $3.6 \pm 1.6$. Most of the study subjects [61\%] were followers of the Ethiopian Orthodox Church, 35.8\% were Protestants, and $3.2 \%$ were Muslim. Concerning their residence, most of the participants were from urban [59.3\%] and peri-urban [20.3\%]. About one-third of the study participants had no formal education, $22 \%$ had primary, $26 \%$ secondary, and $22.7 \%$ tertiary level education. Most of the participants [75.6\%] were unemployed. Over half of the study subjects [56.1\%] had information about nutrition during pregnancy, $40 \%$ of which had this information from health extension workers (Table 1).

Information regarding the birth history of the pregnant women is described in Table 2. More than half of the study subjects were multiparous and had an average of 2-3 children. Close to $80 \%$ of the subjects had $1-3$ children and the remaining $20 \%$ had $4-6$ children. About $35.3 \%$ of the subjects had experienced a birth spacing of 3-4 years (Table 2).

The dietary pattern of the women in the 24 hours preceding the interview showed that cereals, followed by legumes and root and tubers are the most commonly consumed food groups. Considerable percent of study populations consumed Animal Source Food [ASF]. On the other hand, a low consumption of vitamin A- rich vegetables and fruits, and a very low consumption of fruits and vegetables other than those that are vitamin A rich foods were observed (Figure 2).

A surprisingly high number, 31[25.2\%] of pregnant women were fasting the Ethiopian orthodox fasting during pregnancy [Table 3]. The majority of the study subjects, 71[57.8\%] were used to have three meals a day, and about $18 \%$ ate only twice a day. Although coffee is a potential inhibitor of iron absorption, it was consumed by $93.5 \%$ of the subjects among whom $39 \%$ were used to drink 1 to 2 cups per day, and $>10 \%$ of the subjects had coffee consumption exceeding 5 cups a day and coffee was mostly served immediately after a meal [77.2\%]. Similarly, the consumption of tea was experienced by $63.4 \%$ among whom $39.8 \%$ consumed it with or right after a meal.

The health and clinical status of the women are presented in Table 4. About half of the women reported that they had changes in appetite for food during their pregnancy, to be more specific, $72.6 \%$ had a decrease in appetite whereas $27.4 \%$ had experienced an increase in their appetite (Table 4).

Preeclampsia was observed in $10.6 \%$ [ $\mathrm{n}=13]$ women. Among the studied subjects, $35.8 \%$ of the participants were found to be anemic when considering the WHO recommended cut-off of $<11 \mathrm{~g} / \mathrm{dl}$ for anemia and the mean hemoglobin concentration of the anemic women was $9.7 \pm 0.8$. Only $10.6 \%$ of the women
Table 1. Socio-demographic characteristics of third trimester pregnant women at antenatal clinics of Ambo Governmental Health Institutions, West Shoa, Ethiopia, Apr-Aug 2017.

\begin{tabular}{|c|c|c|}
\hline Variables & Frequency (\%), & Mean (SD) \\
\hline Age of the study subjects & & $25.8(5.4)$ \\
\hline Family size per household & & $3.6(1.8)$ \\
\hline \multicolumn{3}{|l|}{ Religion } \\
\hline Orthodox & $75(61.0)$ & -- \\
\hline Protestant & $44(35.8)$ & -- \\
\hline Others & $4(3.2)$ & -- \\
\hline \multicolumn{3}{|l|}{ Urban/rural livelihood } \\
\hline Urban & $73(59.3)$ & -- \\
\hline Peri-urban & $25(20.3)$ & -- \\
\hline Rural & $25(20.3)$ & -- \\
\hline \multicolumn{3}{|l|}{ Marital status } \\
\hline Married & $121(98.4)$ & -- \\
\hline Unmarried & $2(1.6)$ & -- \\
\hline \multicolumn{3}{|l|}{ Ethnicity } \\
\hline Oromo & $107(87.0)$ & -- \\
\hline Amhara & $15(12.2)$ & -- \\
\hline Gurage & $1(0.8)$ & -- \\
\hline \multicolumn{3}{|l|}{ Level of education of the women } \\
\hline Illiterate & $36(29.3)$ & -- \\
\hline Primary school & $27(22.0)$ & -- \\
\hline Secondary school & $32(26.0)$ & -- \\
\hline Tertiary & $28(22.7)$ & -- \\
\hline \multicolumn{3}{|l|}{ Employment status of the women } \\
\hline Employed & $30(24.4)$ & -- \\
\hline Unemployed & $93(75.6)$ & -- \\
\hline Households with access to mass media & $92(74.8)$ & -- \\
\hline $\begin{array}{l}\text { Women having information about nutrition } \\
\text { in pregnancy }\end{array}$ & $70(56.1)$ & -- \\
\hline \multicolumn{3}{|c|}{ Sources of information for women who had information in pregnancy } \\
\hline Radio and television & $9(12.9)$ & -- \\
\hline Community advocacy & $6(8.6)$ & -- \\
\hline Health extension workers & $29(40.0)$ & -- \\
\hline Other & $27(38.5)$ & -- \\
\hline
\end{tabular}

Table 2. Maternal characteristics and pregnancy outcomes of the study subjects at Ambo Governmental Health Institutions, West Shoa, Ethiopia, Apr-Aug 2017.

\begin{tabular}{|c|c|c|}
\hline Variables & Frequency (\%) & Mean (SD) \\
\hline \multicolumn{3}{|l|}{ Pregnancy category } \\
\hline Primiparous & $55(44.7)$ & -- \\
\hline Multiparous & $68(55.3)$ & -- \\
\hline Number of children ever born & -- & $2.34(1.5)$ \\
\hline 01-Mar & $54(79.4)$ & -- \\
\hline 04-Jun & $3(19.1)$ & -- \\
\hline$>6$ & $1(1.5)$ & -- \\
\hline $\begin{array}{l}\text { Gap between previous delivery and current } \\
\text { pregnancy in years }\end{array}$ & -- & $5.03(3.8)$ \\
\hline 01-Feb & $12(17.6)$ & -- \\
\hline 03-Apr & $24(35.3)$ & -- \\
\hline 05-Jun & $15(22.1)$ & -- \\
\hline 7 and above & $17(25)$ & -- \\
\hline
\end{tabular}

have been taking iron or iron folate supplements out of whom $23.1 \%$ took iron and $76.9 \%$ took iron folate supplements. Even more surprising was that very few $[0.8 \%]$ of the women had started to take the supplements during their first trimester.

Dietary diversity score of the pregnant women is presented in Table 5. Majority [26.8\%] of the women consumed only three varieties of food groups and only $2.4 \%$ of the subjects were 
Citation: Assessment of dietary pattern and factors that affect hemoglobin concentration of third trimester pregnant women at Ambo Governmental Health institutions, Ethiopia. 2018;2(2):36-42

consumed all the seven food groups. On the other hand, about $1.6 \%$ of the subjects had consumed one type of food group only (Table 5).

Table 6 presents factors that are associated with hemoglobin concentration of the pregnant women. The level of education of the women was significantly associated with the level of hemoglobin $[\mathrm{p}=<0.00]$ and the coefficient of regression $[\mathrm{B}=.0464]$ showed that there is direct positive relationship between level of education and hemoglobin concentration of the study subjects. Diets of the pregnant women such as legumes and roots and tubers were negatively associated with their hemoglobin concentration as implied by coefficient of regression $[\beta=-.813,95 \% \mathrm{CI}--1.274,-.353], \beta=-0.828,95 \%$ CI- $-1.294,-.363$ ] respectively. In this study, we also observed a positive linear relationship between dietary distribution score and hemoglobin concentration of the women and the coefficient of regression indicated that there is 0.517 unit increase in hemoglobin concentration for every one unit increase in dietary diversity score of the women and this is accompanied by $[95 \%$ CI of $0.203,0.831]$ indicating that we are very confident of the result observed. The average number of the cups of tea and

Table 3. Lifestyle characteristics of third trimester pregnant women attending antenatal clinics of Ambo Governmental Health Institutions, West Shoa, Ethiopia, Apr-Aug 2017.

\begin{tabular}{|c|c|c|}
\hline Variables & Frequency & Percentage \\
\hline Women who had been fasting during pregnancy & 31 & 25.2 \\
\hline \multicolumn{3}{|l|}{ Level of Fasting } \\
\hline Deprivation of animal source food except fish & 14 & 45.2 \\
\hline Deprivation of all animal source foods & 12 & 38.7 \\
\hline $\begin{array}{l}\text { Deprivation of animal source food and fasting until } \\
\text { 3:00 pm }\end{array}$ & 5 & 16.1 \\
\hline \multicolumn{3}{|l|}{ Number of meals per day } \\
\hline Once a day & 3 & 2.4 \\
\hline Twice a day & 22 & 17.9 \\
\hline Three times a day & 71 & 57.8 \\
\hline Four times a day & 24 & 19.5 \\
\hline Five times a day & 3 & 2.4 \\
\hline Breakfast eating habit & 122 & 99.2 \\
\hline Always & 102 & 82.9 \\
\hline Sometimes & 20 & 16.3 \\
\hline Snack taking habit & 74 & 60.2 \\
\hline Always & 22 & 17.9 \\
\hline Sometimes & 52 & 42.3 \\
\hline Coffee/tea consumption over the past two weeks & 115 & 93.5 \\
\hline Coffee Sometimes & 12 & 10.4 \\
\hline Coffee 1 to 2 cups/day & 48 & 41.7 \\
\hline Coffee 3 to 4 cups/day & 41 & 35.7 \\
\hline Coffee 5 and above cups/day & 14 & 12.2 \\
\hline \multicolumn{3}{|l|}{ Timing of coffee intake } \\
\hline Before meal & 3 & 3.5 \\
\hline With meal & 5 & 4.3 \\
\hline Immediately after meal & 95 & 82.6 \\
\hline Approximately 1 hour after meal & 10 & 8.7 \\
\hline Do not know & 1 & 0.9 \\
\hline Tea & 78 & 63.4 \\
\hline Sometimes & 50 & 64.1 \\
\hline 1 to 2 cups/day & 28 & 35.9 \\
\hline \multicolumn{3}{|l|}{ Timing of tea intake } \\
\hline Before meal & 1 & 1.3 \\
\hline With meal & 49 & 62.8 \\
\hline Immediately after meal & 28 & 35.9 \\
\hline
\end{tabular}

Table 4. Health and clinical status of third trimester pregnant women attending antenatal clinics at Ambo Governmental Health Institutions, West Shoa, Ethiopia, Apr-Aug 2017.

\begin{tabular}{|c|c|c|}
\hline Variables & Frequency (\%) & Mean (SD) \\
\hline Appetite changes during pregnancy & $62(50.4)$ & -- \\
\hline Decrease in appetite & $45(72.6)$ & -- \\
\hline Increase in appetite & $17(27.4)$ & -- \\
\hline Pre-eclampsia ${ }^{*}$ during pregnancy & $13(10.6)$ & -- \\
\hline Anemia during pregnancy & $44(35.8)$ & -- \\
\hline Hemoglobin values of the women & -- & $11.3(1.6)$ \\
\hline Hemoglobin value of the anemic women & -- & $9.7(0.8)$ \\
\hline Urban & $26(59)$ & -- \\
\hline Peri-urban & $12(27.3)$ & -- \\
\hline Rural & $13(29.5)$ & -- \\
\hline Hemoglobin value of the non-anemic women & $79(64.2)$ & $12.2(1.0)$ \\
\hline Urban & $47(59.5)$ & -- \\
\hline Peri-urban & $13(16.5)$ & -- \\
\hline Rural & $12(15)$ & -- \\
\hline \multicolumn{3}{|l|}{ Women's blood pressure value } \\
\hline less than $100 / 60 \mathrm{mmHg}$ (hypotensive) & $18(14.6)$ & -- \\
\hline $110 / 65$ to $130 / 85 \mathrm{mmHg}$ (normal BP) & $97(78.9)$ & -- \\
\hline greater than $140 / 90 \mathrm{mmHg}$ (hypertensive) & $8(6.5)$ & -- \\
\hline Women who had been taking medication & $18(14.6)$ & -- \\
\hline Iron supplement & $3(2.4)$ & -- \\
\hline Iron folic acid supplement & $10(8.2)$ & -- \\
\hline Anti-acid & $2(1.6)$ & -- \\
\hline Other & $3(2.4) \mathrm{m}$ & -- \\
\hline \multicolumn{3}{|c|}{ Timing of pregnancy at which iron or iron foliate supplement was started } \\
\hline first trimester & $1(0.8)$ & -- \\
\hline second trimester & $8(6.5)$ & -- \\
\hline third trimester & $4(3.3)$ & -- \\
\hline \multicolumn{3}{|l|}{ The dosage of the supplements being taken ${ }^{\varepsilon}$} \\
\hline One tablet daily $(150 \mathrm{mg})$ & $10(8.2)$ & -- \\
\hline Two tablets daily (300 mg) & $1(0.8)$ & -- \\
\hline Three tablets daily $(450 \mathrm{mg})$ & $2(1.6)$ & -- \\
\hline \multicolumn{3}{|c|}{$\begin{array}{l}\text { ¥: Persistent swelling of feet, hands or face, increased breathlessness (especially } \\
\text { on routine activity), headaches, blurring of vision, fever (temperature }>38^{\circ} \mathrm{C} \text { ) and } \\
\text { high colored urine in the past two weeks } \\
£ \text { : iron or iron foliate }\end{array}$} \\
\hline
\end{tabular}

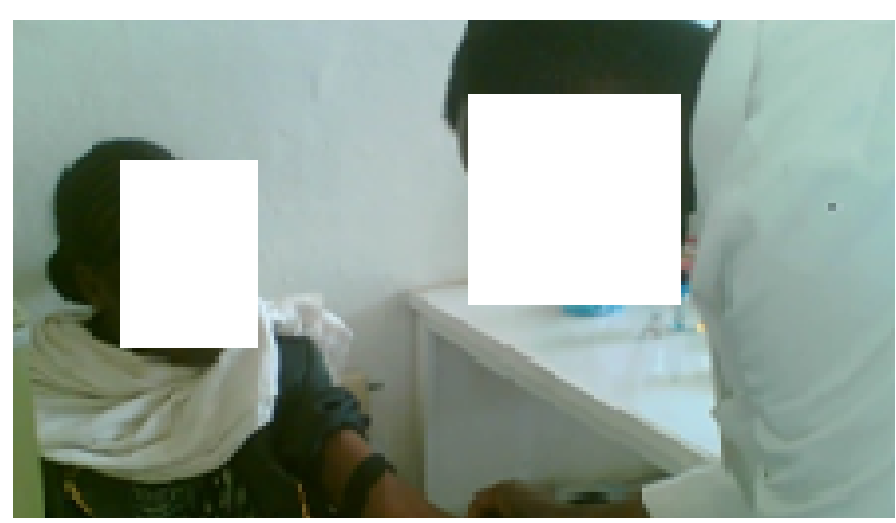

Figure 1. Making venipuncture in the laboratory for hemoglobin determination.

coffee taken per day is also statistically negatively associated with hemoglobin concentration of the women with $[\mathrm{p}=0.003]$ and $[\beta=-0.476]$ for tea and $[p=0.020]$ and $[\beta=-0.290]$ for coffee with $95 \%$ CI of $[-0.792,-0.161]$ and $[-0.532,-0.047]$ respectively (Table 6).

\section{Discussion}

Micronutrient deficiencies, especially iron deficiency and anemia, are common during pregnancy. In this study, maternal 


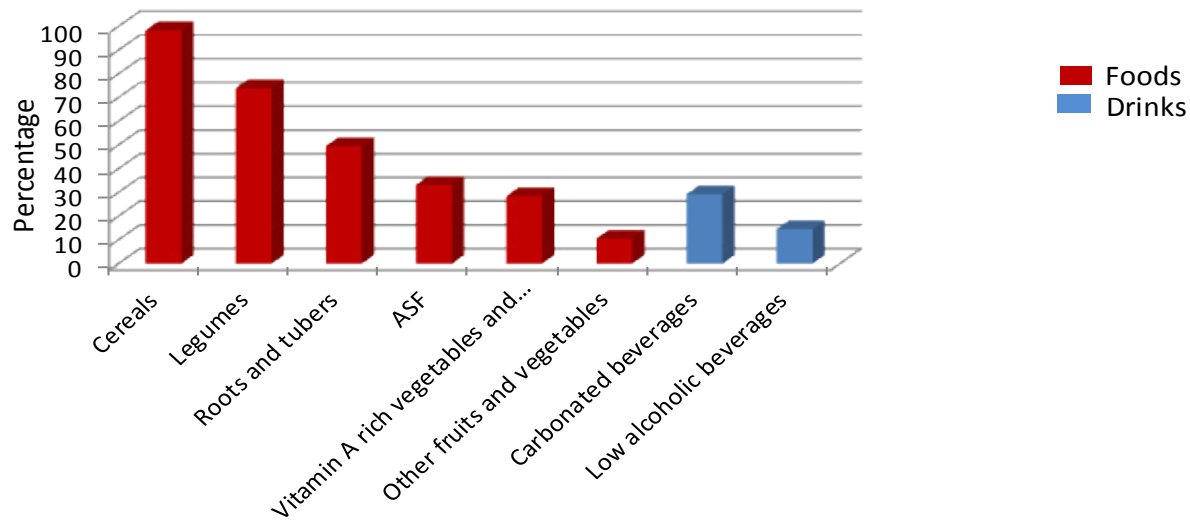

Figure 2. Dietary pattern (24 hr recall) of third trimester pregnant women attending antenatal clinics at Ambo Governmental Health Institutions, West Shoa, Ethiopia, Apr-Aug 2017.

Table 5. Dietary diversity score of third trimester pregnant women attending antenatal clinics at Ambo Governmental Health Institutions, West Shoa, Ethiopia, Apr-Aug 2017.

\begin{tabular}{|c|c|c|}
\hline Diet score & quency & Percent \\
\hline 1.00 & 2 & 1.6 \\
\hline 2.00 & 12 & 8.9 \\
\hline 3.00 & 34 & 26.8 \\
\hline 4.00 & 26 & 21.1 \\
\hline 5.00 & 31 & 25.2 \\
\hline 6.00 & 15 & 12.2 \\
\hline 7.00 & 3 & 2.4 \\
\hline
\end{tabular}

Table 6. Factors associated with hemoglobin concentration of third trimester pregnant women attending antenatal clinics at Ambo Governmental Health Institutions, West Shoa, Ethiopia, Apr-Aug 2017.

\begin{tabular}{|c|c|c|c|c|c|}
\hline \multirow[b]{2}{*}{ Variables } & \multicolumn{5}{|c|}{$95 \% \mathrm{Cl}$ for $\mathrm{B}$} \\
\hline & B & $\begin{array}{l}\text { Lower } \\
\text { Bound }\end{array}$ & $\begin{array}{l}\text { Upper } \\
\text { Bound }\end{array}$ & P-value & Adj. $R^{2}$ \\
\hline Level of education & 0.464 & 0.241 & 0.687 & $.001^{* *}<$ & -- \\
\hline \multirow{2}{*}{ Parity } & \multirow{2}{*}{-0.096} & \multirow{2}{*}{-0.274} & \multirow{2}{*}{0.081} & \multirow{2}{*}{0.284} & -- \\
\hline & & & & & -- \\
\hline Legumes & -0.813 & -1.274 & -0.353 & $.001^{* *}$ & \\
\hline Cereals & 0.607 & 0.187 & 1.026 & $.005^{\star *}$ & 0.396 \\
\hline Roots and Tubers & -0.828 & -1.294 & -0.363 & $.001^{* *}$ & -- \\
\hline ASF & -0.581 & -1.084 & -0.078 & $.024^{*}$ & -- \\
\hline DDS & 0.517 & 0.203 & 0.831 & $.001^{* *}$ & -- \\
\hline Carbonated drink & 1.005 & 0.454 & 1.555 & $.001^{* *}<$ & -- \\
\hline $\begin{array}{l}\text { Average number of cups } \\
\text { of tea taken/day }\end{array}$ & -0.476 & -0.792 & -0.161 & $.003^{\star *}$ & -- \\
\hline $\begin{array}{l}\text { Average number of cups } \\
\text { of coffee taken /day }\end{array}$ & -0.29 & -0.532 & -0.047 & $.020^{*}$ & -- \\
\hline Number of meals per day & 0.389 & 0.056 & 0.723 & $.023^{*}$ & -- \\
\hline
\end{tabular}

socio-demography and dietary pattern were associated with hemoglobin.

About one-third of the pregnant women in this study were anemic. Several reasons can explain this finding. Most studies indicated that maternal malnutrition contributes to the prevalence of anemia [1,2]. For instance, the dietary pattern of the pregnant women showed that the diets were predominantly plant-based with little consumption of ASF. Although cereal consumption was positively associated with maternal hemoglobin concentrations [ $p=0.005]$, consumption of legumes was negatively associated with maternal hemoglobin in this study. Hence, it is recognized that, non-heme [plant] iron, unlike that of heme is susceptible to the effects of chelating agents such as phytate and polyphenols and is thus of lower bioavailability [4]. Likewise, this is also supported by this study in that consumption of legumes was found to be important predictor of maternal hemoglobin concentration justifying poor iron bioavailability. Besides the high coffee and tea consumption by the pregnant women, especially for those who consumed them right before or after a meal, is likely to further compromise the bioavailability of minerals like iron. Increased tea consumption reduces iron absorption [13]. Indeed, consumption of tea in this study was an important predictor of maternal hemoglobin. In contrast, sources of iron absorption enhancers such as vitamin-A rich fruits and vegetables, ascorbic acid rich foods such as citrus fruits were rarely consumed. Unlike ASFs, fruits were available in the community, but did not constitute the food basket of the pregnant women possibly because it is more expensive than staples like cereals and legumes or possibly due to the monotonous food habit of the community. This suggests that education on nutrition during pregnancy is required, especially given the fact that significant proportions of the pregnant women have reported not having any information on optimal practices during pregnancy. Such nutritional education should recommend consumption of a diversified diet, especially given the fact that DDS and number of meals were important predictors of hemoglobin concentration in this study.

Apart from maternal dietary pattern, there are also other maternal factors that showed association with hemoglobin concentration and these include; level of education and the number of meals consumed by the pregnant women. Optimal weight gain during pregnancy can help mothers achieve favorable pregnancy outcomes and can contribute to the wellbeing of the fetus as well. The association of level of education with hemoglobin concentration in this study is in line with previous findings [7] and may indicate that women with better education may have better understanding of optimal feeding during pregnancy. Therefore, women's education must be addressed, particularly during adolescence. Studies also indicated that multiparty may lead to anemia by reducing maternal iron reserves at every pregnancy and by causing blood loss at each delivery [12]. However, no statistical significance was obtained between anemia and parity in this study. This may be due to two reasons; first, more than $79 \%$ of the multiparous women in this study have only 1-3 children among whom more than $80 \%$ of the women had 
experienced birth spacing of greater than three years. Hence, repeated bleeding during delivery would be minimal. Secondly, compared to primiparous women most multiparous women had been taking iron or iron folate supplements. Yet, the combined effect of the factors discussed above resulted in $35.8 \%$ of anemia prevalence in this study community which is relatively higher compared to the studies from African countries like Nigeria [20]. Although it is less than the prevalence of anemia in Galgal gibe of Ethiopia [9] it is greater than the report from South east Ethiopia [18]. This might be due to geographical differences, dietary patterns and other confounding factors such as communicable and non-communicable diseases.

The timing of prenatal intakes or supplementations of specific micronutrients is vital as pregnancy is characterized by different stages that represent a continuum, up to lactation and beyond and hence applying to different requirements. Consequently, nutrition during pregnancy might have long-lasting effects on the well-being of the mother and the fetus and may further influence the health of the baby at a later age $[12,14]$. Nonetheless, very few subjects in this study had been taking supplements and only $0.8 \%$ of them started to take iron folic acid supplement during first trimester. Even though most women have good antenatal follow up they quit taking the supplements and this requires further study to understand the cause of the problem. In the mean time, appropriate strategies to reduce anemia through synergistic effects of improved food intake, nutrition education, and supplementation is crucial.

\section{Limitation of the study}

Some of the study participants did not accurately recall what they ate in the past two weeks, and this might have affected the results from the two-week food frequency.

\section{Conclusion}

About one-third of the pregnant women in this study were anemic and this was due to dietary pattern of the pregnant women. The diet of the pregnant women was predominantly plant-based with little consumption of fruits and vegetables. Tea and coffee consumption were an important predictor of maternal hemoglobin concentration. In addition, level of education and number of meals per day were strongly associated with hemoglobin concentration. Even though the women had good antenatal follow up they do not have good adherence to iron or iron-folate supplements. Diversification of the diet increases hemoglobin concentration implying a reduced occurrence of anemia. Maternal micronutrients requirement increases due to increased physiological demand to enable the development of the fetus. Pregnant women should reduce the intake of foods like roots and tubers that simply fill their satiety, but do not supply adequate nutrients for maternal weight gain and fetal development or these foods must be complemented with other nutrient rich foods. It is imperative to avoid consumption of tea and coffee whenever possible or decrease the amount consumed per day to prevent anemia. In addition, foods like legumes should not be taken in bulk as they impair bioavailability of iron. Women should seek ANC as early as they realize that they are pregnant and should adhere to recommended supplements like iron/iron-folic acid to prevent both mother and neonatal anemia and hence prevent the associated adverse effects on child development and cognition. Further research is needed to investigate why adherence to recommended micronutrient supplement is low. It is also vital for ANC attendants to check the hemoglobin status of pregnant women regularly and follow up the proper supplementation and adherence. In addition, it is also crucial to make follow up on their gestational weight gain. The health educators must also teach the mothers about adequate nutrition throughout pregnancy.

\section{Declarations}

\section{Ethical clearance and approval}

The proposal was presented to Addis Ababa University ethical review board for approval and the research was started after obtaining ethical clearance from Oromia regional health bureau. The benefit of the study was explained to health department of West Shoa zone, Ambo woreda health bureau, Ambo hospital and Ambo health center administrative officials. A written and signed consent was obtained from pregnant women during the study period.

\section{Consent to publish}

Not applicable

\section{Availability of data and materials}

The data sets used and analyzed during this study period are available from Menelik II Health Science College, Addis Ababa, Ethiopia when reasonably required by requesting $\mathrm{Mr}$. Kefiyalew Jote.

\section{Competing interests}

No competing interest.

\section{Funding}

This research has been funded by Addis Ababa University, Ethiopia.

\section{Author's contributions}

KJ proposed the idea, developed the full proposal, and directly participated in the recruitment of the study subjects and data collectors. He also directly involved in the data collection process, supervised the data collectors and analyzed the data collected and made the final write up. EG provided more support in the data analysis and write up of the final version. All authors read and agreed on the manuscript.

\section{Acknowledgements}

We are very grateful to Ambo Hospital, Ambo health center and Awaro health center antenatal clinic nurses, clinicians and medical laboratory professionals for their indispensible cooperation during the data collection. Our special gratitude will also go to Ambo hospital cesarean section case team for their indispensible cooperation during this study period.

\section{References}

1. Badham J, Zimmermann MB, Kraemer K. The guide book: Nutritional Anemia. Sight and Life Press. 2007; ISBN 3-906412-35-0.

2. Kalaivani K. Prevalence and consequences of anemia in pregnancy. Indian J Med Res. 2009;130:627-633. 
Citation: Assessment of dietary pattern and factors that affect hemoglobin concentration of third trimester pregnant women at Ambo Governmental Health institutions, Ethiopia. 2018;2(2):36-42

3. Muthayya S. Maternal nutrition and low birth weight-what is really important? Indian Journal of Medical Research. 2009; 130:600-608.

4. Brune, M, Rossander-Hulten L, Hallberg L, et al. Iron absorption from bread in humans: inhibiting effects of cereal fiber, phytate and inositol phosphates with different numbers of phosphate groups. J. Nutr. 1992;122:442-449.

5. Dallman PR, Siimes MA, Stekel A. Iron deficiency in infancy and childhood. The Am J of Nutr. 1980;33:86-118.

6. Ahmed F, Mahmuda I, Sattar A, et al. Anemia and vitamin A deficiency in poor urban pregnant women of Bangladesh. Asia Pac J Clin Nutr. 2003;12(4):460-6.

7. Sharma P., Mehta S, Nagar R. Prevalence of anemia and soci-demographic factors associated with anemia among pregnant women attending antenatal Hospital in Jaipur city. IOSR-JPBS. 2013;6(3):01-05.

8. Kefiyalew F, Zemene E, Asres Y, et al. Anemia among pregnant women in Southeast Ethiopia. Prevalence, severity and associated risk factors. BMC Research Notes. 2014;7:771.

9. Getachew M, yewhualaw D, Taffess K, et al. Anemia and associated risk factors among pregnant women in Gilgel Gibe dam area, Southeast Ethiopia. Parasites and vectors. 2012;5:296.

10. Upadhyaya C. Mishra S., Ajmera P, et al. Serum iron, copper and zinc status in maternal and cord blood. Indian $\mathrm{J}$ of Clini Bioche. 2004;19(2):48-52.

11. Alene KA, Dohe AM. Prevalence of anemia and associated factors among pregnant women in an urban area of Eastern Ethiopia. 2014 http://dx.doi.org/10.1155/2014/561567

12. Karauglul L, Pehlivan E, Egri M, et al. The prevalence of nutritional anemia in pregnancy in an east Antolian province, Turkey. BMC public health. 2010;10:329.

13. Speedyp AW. Global Production and Consumption of Animal Source Foods. J Nutr, 2003;133(Suppl 2):4048-53.

14. Sahu KK, Idris MZ, Agarwal M, et al. Umbilical cord blood nutrients among low birth weight and normal birth weight babies in primary health care setup in Lucknow, India. $\mathrm{J}$ of Bio and Sci Opinion. 2013;1(4).

15. Shao J, Lou J, Rao R, et al. Maternal serum ferritin concentration is positively associated with new born iron stores in women with low ferritin status in late pregnancy. J.nutr. 2012;142:2004-09.

16. Black RE. Micronutrient in pregnancy. Br J Nutr. 2001; 85:193-197.

17. Gambling L, Danzeisen R, Gaie S, et al. Effect of iron deficiency on placental transfer of iron and expression of iron transport proteins in vivo and in vitro. Biochem $\mathrm{J}$. 2001;356(3):883-9.

18. Garg A, Kashyap S. Effect of counseling on nutritional status during pregnancy. Indian J Pediatr. 2006;73(8): 687-692.

19. Salahat MA, Ibrahim AI. Prevalence of anemia among Jordanian pregnant women and the effect of early pregnancy on alkaline phosphatase activity. Jord $\mathrm{J}$ of Bio Sci. 2012;5(1):65-70.

20. Erhabor O, Isah A, Isaac IZ, et al. Iron deficiency anemia among antenatal women in Sokoto, Nigeria. British Journal of Medical and Health Sciences. 2013;1(4):47-55.

21. UNICEF/WFP/WHO. Asia Regional Workshop on the reduction of stunting through improvement of complementary feeding and maternal nutrition. 2010.

\section{*Correspondence to:}

Kefiyalew J

Kotebe Metropolitan University

Addis Ababa,

Ethiopia

Tel: +251 116600921

E-mail: naftan2006@yahoo.com 